\title{
Предварительные результаты эксперимента «FENICS-2019»
}

\author{
Шевцов А.Н. ${ }^{1}$, Баранник М.Б. ${ }^{2}$, Ганнибал А.Е. ${ }^{3}$, Горохов О.Ю. ${ }^{4}$, Жамалетдинов А.А. ${ }^{1,5}$, \\ Ивонин В.В. ${ }^{2}$, Казаков Б.В. ${ }^{6}$, Колобов В.В. ${ }^{2}$, Короткова Т.Г. ${ }^{1}$, Скороходов А.А. ${ }^{1}$, Стрельцов С.В. ${ }^{7}$ \\ ${ }^{1}$ Геологический институт КНЦ РАН, Anamumbl, anshev2019-01@mail.ru

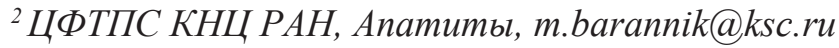 \\ ${ }^{3}$ Геофизическая служба РАH, Anamumbl,abd.zham@mail.ru \\ ${ }^{4}$ ОАО «СО ЕЭС» Кольское РДУ, Мурмаши, ougorohov@kola.so-ups.ru \\ ${ }^{5}$ СПбФ ИЗМИРАН, С.-Петербург, abd.zham@mail.ru \\ ${ }^{6}$ ОАО «МРСК Северо-Запада» «Колэнерго», Мурмаши, ougorohov@kola.so-ups.ru \\ ${ }^{7}$ ФСК «Северо-Запад», Санкт-Петербург, mes1@sz.fsk-eеs.ru
}

Аннотация. Работа посвящена предварительным результатам эксперимента FENICS (Fennoscandian Electrical conductivity from Natural and Induction Controlled Sources soundings) по изучению глубинной электропроводности литосферы Фенноскандинавского щита на территории Кольского полуострова и Карелии с использованием заземленных участков промышленных линий электропередачи. В рамках эксперимента FENICS-2019 измерения выполнялись рядом отечественных и зарубежных исследователей. Измерения с контролируемым источником выполнялись в диапазоне частот от 0.1 до 194.2 Гц, при удалении точки измерений от центра источника на расстоянии от 100 до 875 км. В качестве источника электромагнитного поля использовались заземленные участки промышленных линий электропередачи с генератором тока «Источник ЭНЧ» мощностью до 200 кВт (разработка ЦЭС КНЦ РАН, изготовитель ПГИ РАН). Измерения ГИ КНЦ РАН выполнены с помощью отечественных станций VMTU-10 (ООО «ВЕГА», г. Санкт-Петербург). Предварительные результаты представлены с использованием результатов предыдущих экспериментов серии FENICS в виде вертикальных квази-двумерных разрезов по трем субмеридиональным профилям, и набора горизонтальных квази-трехмерных разрезов для глубины 3, 10, 20 и 100 км, полученных интерполяцией результатов решения одномерной обратной задачи в точках наблюдения.

Ключевые слова: частотное зондирование, электропроводность, кажущееся сопротивление, литосфера, Фенноскандинавский щит.

\section{Preliminary Results of the FENICS-2019 Experiment}

\author{
Shevtsov A.N. 1, Barannik M.B. ${ }^{2}$, Hannibal A.E. ${ }^{3}$, Gorokhov O.Yu. ${ }^{4}$, Zhamaletdinov A.A. ${ }^{1,5}$, \\ Ivonin V.V. ${ }^{2}$, Kazakov B.V. ${ }^{5}$, Kolobov V.V. ${ }^{2}$, Korotkova T.G. ${ }^{1}$, Skorokhodov A.A. ${ }^{1}$, Streltsov S.V. ${ }^{7}$ \\ ${ }^{1}$ GI KSC RAS, Apatity, anshev2019-01@mail.ru \\ ${ }^{2}$ CNPE KSC RAS, Apatity, m.barannik@ksc.ru \\ ${ }^{3}$ Geophysical service RAS, Apatity, abd.zham@mail.ru \\ 4OJSC "SO UES” Kola RDU, Murmashi, ougorohov@kola.so-ups.ru \\ ${ }^{5}$ SPbF IZMIRAN, St.Petersburg, abd.zham@mail.ru \\ ${ }^{6}$ JSC IDGC of the North-West Kolenergo, Murmashi,ougorohov@kola.so-ups.ru \\ ${ }^{7}$ JSC IDGC of the North-West Kolenergo, Saint-Petersburg,mes1@sz.fsk-ees.ru
}

\begin{abstract}
The article is devoted to a preliminary results of an experiment FENICS (Fennoscandian Electrical conductivity from Natural and Induction Controlled Sources soundings) for studying the deeping electrical conductivity of the lithosphere of the Fennoscandian Shield on the Kola Peninsula and Karelia using grounded sections of industrial power lines. As part of the FENICS-2019 experiment, measurements were performed by several domestic and foreign researchers. Measurements with a controlled source were performed in the frequency range from 0.1 to $194.2 \mathrm{~Hz}$, when the measurement point was removed from the center of the source at a distance of 100 to $875 \mathrm{~km}$. As the source of the electromagnetic field, grounded sections of industrial power lines with current generator «Source ULF» with a capacity of up to $200 \mathrm{~kW}$ were used (developed by the Center for the North Power Engineering, Kola Science Center and Polar Geophysical Institute of Russian Academy of Sciences). The measurements of the GI KSC RAS were carried out with the domestic stations VMTU-10 (VEGA LLC St. Petersburg). Preliminary results are presented with the use of data from previous FENICS experiments in the form of vertical quasi-two-dimensional sections along three
\end{abstract}


submeridional profiles, and a set of horizontal quasi-three-dimensional sections for depths of 3, 10, 20, and $100 \mathrm{~km}$ obtained by interpolating the results of solving of a one-dimensional inverse problem at observation points.

Key words: frequency sensing, electrical conductivity, apparent resistance, lithosphere, Fennoscandian Shield.

\section{Первичные данные}

Обработка данных и интерпретация выполнена на основе результатов измерений, полученных в экспериментах «FENICS-2007», «FENICS-2014» и «FENICS-2019». Для этого выбраны профили 1, 2, 3 показанные на рисунке 1.

Качество регистрации сигналов проиллюстрировано на рисунке 2 на примере наблюдений, выполненных в точке Сямозеро на удалении 752 км от источника, от центра линии Л-401.

Из рисунка 2 можно видеть, что наиболее отчетливо, с превышением над шумами до 1-го порядка, записаны сигналы на главных сопряженных компонентах Еу (восток-запад) и Нх (север-юг). Уверенная регистрация сигналов наблюдается в диапазоне частот 1-100 Гц.

\section{Учет статических искажений}

Научной новизной методики обработки первичных данных в эксперименте «FENICS-2019» явилось применение способа количественного учета статических искажений. Основа разработанного способа учета статических искажений - использование измерений полного горизонтального магнитного поля в пределах действия условий дальней зоны квазистационарного (волнового) приближения. Теоретическое обоснование базируется на уравнениях поля заземленного диполя, ориентированного вдоль оси $X$ (Ваньян, 1965). Уравнения для напряженности продольного относительно оси диполя АВ электрического поля $E_{x}$, поперечного магнитного поля $H_{y}$ и входного импеданса $Z_{x y}$ имеют вид (Жамалетдинов, 2012, 2019):

$$
\begin{gathered}
E_{x}=\frac{I L_{A B}\left(3 \cos ^{2} \theta-2\right)}{2 \pi r^{3}} \rho, \mathrm{B} / \mathrm{M} ; \\
H_{y}=\frac{I L_{A B}\left(3 \cos ^{2} \theta-2\right)}{2 \pi r^{3} \sqrt{\omega \mu_{0}}} \sqrt{\rho} \mathrm{A} / \mathrm{M} ; \\
\left|Z_{x y}\right|=\left|\frac{E_{x}}{H_{y}}\right|=\sqrt{\omega \mu_{0} \rho} \mathrm{OM} \times \mathrm{M} .
\end{gathered}
$$

Из выражений (1-3) видно, что удельное электрическое сопротивление нижнего полупространства в поле заземленного электрического диполя можно определять как из электрического (1), так и из магнитного (2) полей отдельно и из их соотношений, если использовать входной импеданс (3). Разница между соотношениями (3) и (1)-(2) заключается в том, что напряженность электрического поля (1) линейно зависит от электросопротивления, тогда как напряженность магнитного поля (2) и входной импеданс (3) зависят от электрического сопротивления нижнего полупространства под квадратным корнем.

Еще одна особенность заключается в том, что магнитный датчик изолирован от земли. По этой причине интенсивность измеряемого магнитного поля не подвержена влиянию статического сдвига, а кажущееся сопротивление, рассчитанное по магнитному полю, может использоваться в качестве меры для количественной оценки искажений статического сдвига, влияющих на электрические и импедансные нормировки поля (Жамалетдинов, 2019, Шевцов 2019).

Кажущееся удельное сопротивление рассчитывается для модулей полных векторов горизонтальных магнитных и электрических полей и импедансному отношению по формулам (Шевцов, 1989, 2019).

Полученные кривые кажущегося сопротивления по импедансу в поле контролируемого источника дополнялись значениями кажущегося сопротивления по импедансу поля естественного происхождения, измеренного в тех же точках (АМТ - поле) в более широком частотном диапазоне. 


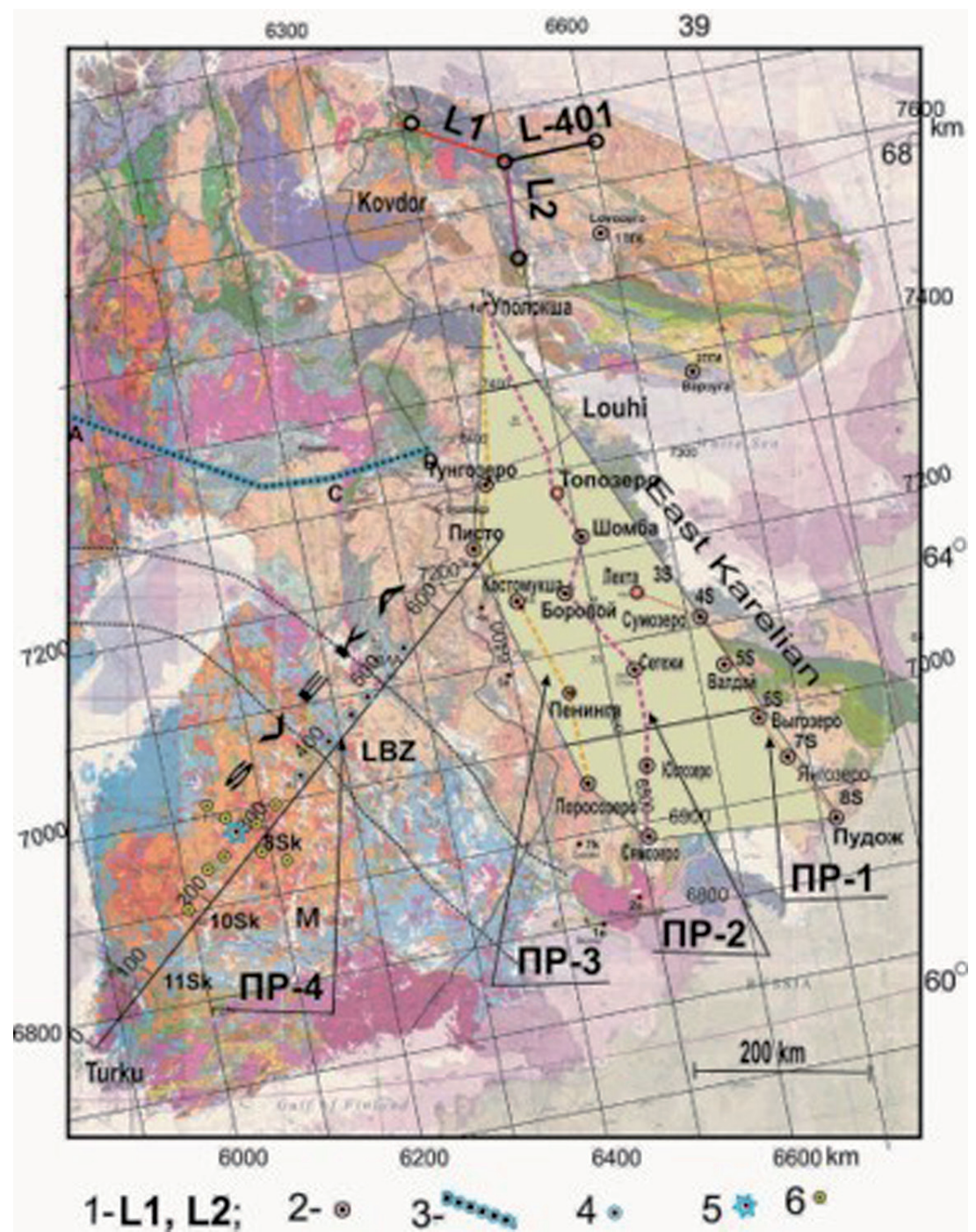

Pис. 1. Схема расположения питающих линий (L1, L2 и L-401) и пунктов зондирования в экспериментах «FENICS-2019» (профиль ПР-1), «FENICS-2014» (профиль ПР-2) и «FENICS-2007» (профиль ПР-3). Профиль ПР-4 («SVEKA»).

1 - заземленные промышленные линии электропередачи L1 (Л-403), L2 (Л-153/154) и L-401; 2 - точки зондирования на территории России (ГИ и ЦЭС КНЦ РАН, г. Апатиты) на профилях 1-3; 3 - точки зондирования на территории Швеции (Университет Лулео) и Финляндии (Геологическая служба Финляндии, г. Рованиеми), выполненные вместо плановых точек на профиле SVEKA; 4 - плановые точки зондирования на профиле SVEKA; 5 - выполненные ранее точки зондирования на территории Финляндии (Университет Оулу); 6 - пункты частотных зондирований CSAMT, выполненные ГИ КНЦ РАН на профиле SVEKA в 1997 г. (Zhamaletdinov et al., 1998, 2002). На рисунке 1, наряду с прямоугольной координатной сеткой Гаусса-Крюгера приведена географическая сетка координат и даны соответствующие обозначения в километрах и в градусах. Бежевым фоном выделена область для квази-3D инверсии.

Fig. 1. Layout of supply lines (L1, L2 and L-401) and sensing points in the experiments "FENICS-2019" (profile PR-1), "FENICS-2014" (profile PR-2) and "FENICS-2007" (profile PR-3). Profile PR-4 ("SVEKA").

1 - grounded industrial power lines L1 (L-403), L2 (L-153/154) and L-401; 2 - sensing points on the territory of Russia (GI and CES KSC RAS, Apatity) on profiles 1-3;3 - sounding points on the territory of Sweden (Lulea University) and Finland (Geological Survey of Finland, Rovaniemi), performed instead of the planned points on the SVEKA profile; 4 - planned sounding points on the SVEKA profile; 5 - previously performed sounding points in Finland (University of Oulu); 6 - CSAMT frequency sounding points performed by the GI KSC RAS on the SVEKA profile in 1997 (Zhamaletdinov et al., 1998, 2002). Fig. 1, along with the rectangular Gauss-Krueger coordinate grid, shows the geographical coordinate grid and gives the corresponding notation in kilometers and degrees. The beige background marks the area for quasi-3D inversion. 


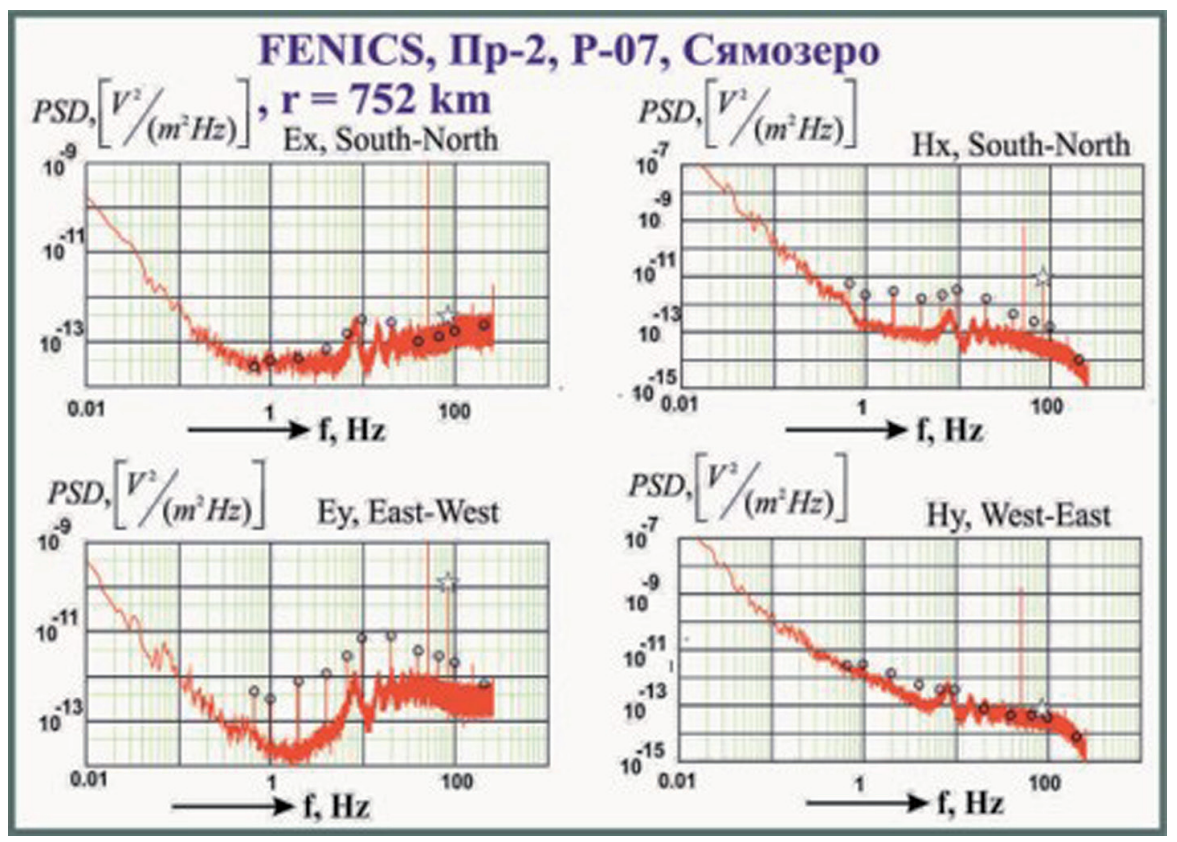

Рис. 2. Графики спектральной плотности мощности по результатам регистрации сигналов в точке Сямозеро на удалении 752 км от источника.

Fig. 2. Graphs of the power spectral density according to the results of the registration of signals at the Syamozero point at a distance of $752 \mathrm{~km}$ from the source.

\section{Результаты инверсии кривых кажущегося сопротивления}

После коррекции за статические искажения выполнялась инверсия с помощью дифференциальной трансформации Молочного-Вьета и путем решения одномерной обратной задачи частотного зондирования с дипольным источником методом сопряженных градиентов. Далее, на следующем

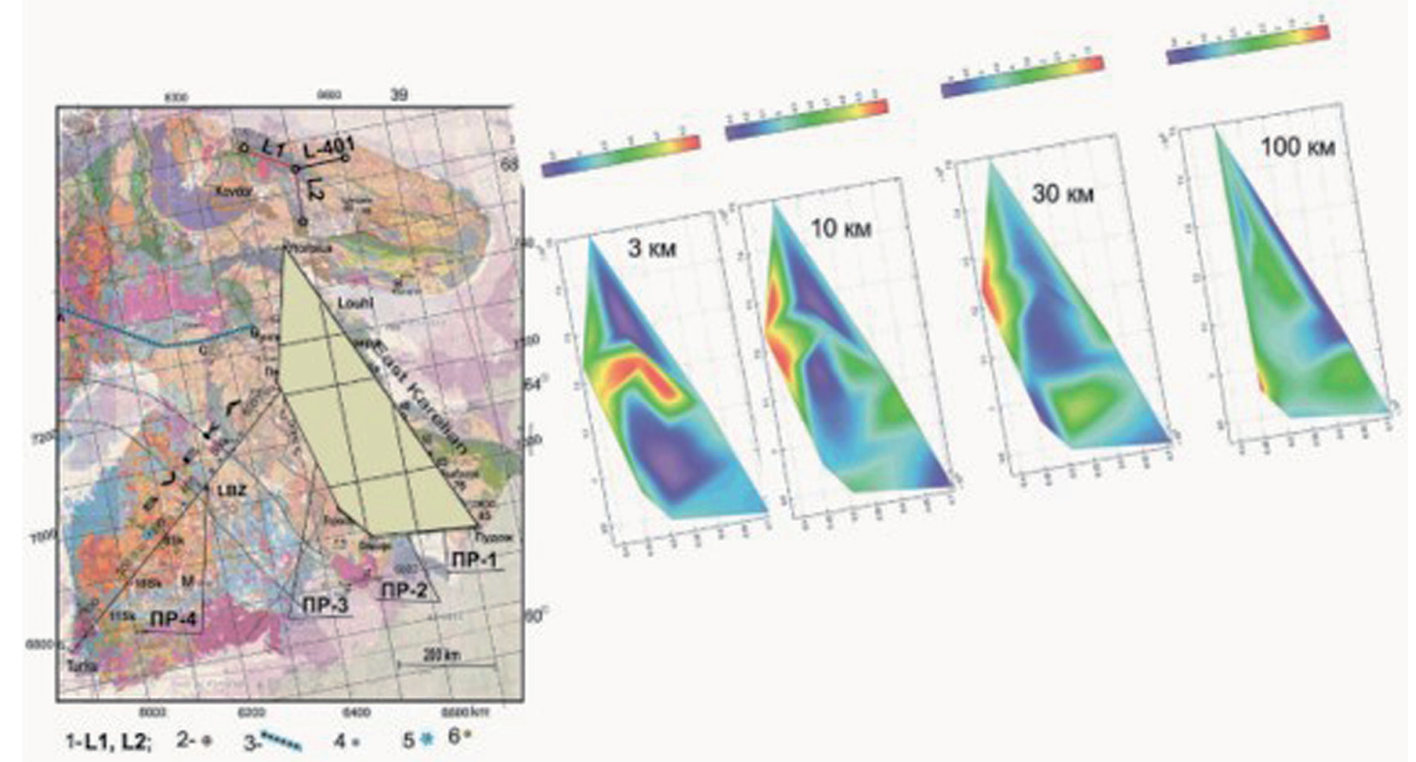

Рис. 3. Квази-трехмерная модель электропроводности литосферы на основе одномерных инверсий по методу сопряженных градиентов. Бежевым фоном на левом рисунке показана площадь построения квази-3D модели, показанной справа для глубин 3, 10, 30 и 100 км.

Fig. 3. Quasi-three-dimensional model of the electrical conductivity of the lithosphere based on one-dimensional inversions by the method of conjugate gradients. The beige background in the left figure shows the construction area of the quasi-3D model shown on the right for depths of 3, 10, 30 and $100 \mathrm{~km}$. 
этапе производится построение квази-трехмерной модели на основе построенных квази-двухмерных разрезов. На первом этапе квази-трехмерная модель представляется в виде серии горизонтальных срезов. Результат такой обработки приведен ниже на рисунке 3.

Модель характеризуется относительно небольшим диапазоном изменений удельного электрического сопротивления в пределах $10^{4}-10^{5}$ Ом м для глубин 3, 10, 30 и 100 км. С глубиной контрастность разреза уменьшается, что свидетельствует о гомогенизации вещества. Представленная модель является самым первым приближением. Для ее доработки требуется проведение большого объема расчетно-методических и вычислительных работ, предусмотренных на третий этап реализации проекта 18-05-00582.

Работа выполнена при финансовой поддержке РФФИ, проект № 018-05-00528, а также в рамках госзадания Министерства образования и науки РФ - тема ГИ КНЦ РАН № 0226-2019-0052 и тема ЦЭС КНЦ РАН № 0226-2019-0067.

\section{Литература}

1. Ваньян Л.Л. Основы электромагнитных зондирований. М. Изд-во: Недра. 1965. 107 с.

2. Жамалетдинов А.А. Способ количественного учета статических искажений по магнитному полю контролируемого источника CSAMT // Наука и технологические разработки. Вып. «Методические разработки для электромагнитных зондирований с управляемыми источниками». 2019. Т. 98. № 4. С. 5-18. DOI: $10.21455 /$ std2019.4-1.

3. Шевцов А.Н. О некоторых способах нормировки и трансформации результатов электромагнитных зондирований // Глубинные геоэлектрические исследования с использованием промышленных линий электропередач. Апатиты. 1989. С. 90-95.

4. Шевцов А.Н. Обработка и интерпретация данных глубинного частотного зондирования в комплексе с данными АМТЗ в эксперименте «Мурман-2018» // Наука и технологические разработки. Вып. «Методические разработки для электромагнитных зондирований с управляемыми источниками». 2019. Т. 98. № 4. C. 19-33. DOI: 10.21455/std2019.4-2.

5. Zhamaletdinov A.A., Shevtsov A.N., Tokarev A.D., Korja T., Pedersen L. Experiment on the Deep Frequency Sounding and DC Measurements in the Central Finland Granitoid Complex // Electromagnetic Induction in the Earth. 14-th Workshop in Sinaia (Romania). 1998. P. 83.

6. Zhamaletdinov A.A., Shevtsov A.N., Tokarev A.D. and Korja T. Electromagnetic Frequency Sounding of the Earth Crust beneath the Central Finland Granitoid Complex // Izvestiya, Physics of the Solid Earth. V. 38. N. 11. P. 954-967. (Translated from Fizika Zemli. N. 11. 2002. P. 54-68). 